\title{
Modèles prévisionnels de comportement élastique tridimensionnel pour les bois feuillus et les bois résineux
}

\author{
D. GUITARD, F. EL AMRI \\ I.N.P.L., Laboratoire d'Energétique et de Mécanique théorique et appliquée, \\ B.P. 850. F54011 Nancy Cedex
}

\begin{abstract}
Résumé
Une banque de données informatisée, reproduite ici. comprend les propriétés physiques, masse volumique $\rho$, et taux d'humidité $H$, et les propriétés élastiques tridimensionnelles de 80 bois. Une analyse statistique a permis d'établir des régressions multiples significatives entre ces deux groupes de paramètres; elle conduit à la proposition de deux modèles prévisionnels de comportement élastique tridimensionnel l'un pour les bois feuillus et l'autre pour les bois résineux, et permet de prévoir de façon satisfaisante la matrice complète des complaisances élastiques pour un bois dont la masse volumique $\rho$ est connue à un taux d'humidité voisin de $12 \mathrm{p} .100$. L'efficacité du modèle bois feuillus est discutée à partir de résultats expérimentaux obtenus sur le bois d'un même hêtre par neuf laboratoires.
\end{abstract}

Mots clés : Elasticité, anisotropie, feuillus, résineux, modèles prévisionnels, masse volumique.

\section{Introduction}

La loi de comportement élastique, de complexité minimale, apte à rendre compte de certaines spécificités du comportement mécanique du matériau bois, implique de prendre en considération la forte anisotropie de ce matériau. Ceci a conduit, depuis fort longtemps (HEarmon, 1948) de nombreux chercheurs à développer des moyens expérimentaux variés, complexes et souvent coûteux, en vue d'identifier les nombreuses constantes élastiques ( 9 au minimum) nécessaires pour rendre compte du comportement élastique tridimensionnel du bois considéré, les expériences étant à renouveler pour les diverses essences.

Les auteurs présentent ici, une banque de données, relative à certaines caractéristiques élastiques et physiques de bois feuillus et de résineux extraites pour une part de sources bibliographiques variées, et pour certaines directement de travaux de notre laboratoire (Seichepine, 1980 ; Ben Farhat, 1985).

Un traitement statistique confirme la masse volume $\rho$ comme paramètre physique fortement explicatif de la variabilité totale des caractéristiques élastiques inter et intra essences.

Les résultats de recherche de régressions simples ou multiples, conduisent à la proposition de deux modèles prévisionnels pour le comportement mécanique élastique l'un pour les bois «feuillus » et l'autre pour les bois « résineux». Le présent travail complète l'excellent document publié en 1973 par J. Bodig et J.R. Goodmann, en apportant de nouvelles données bibliographiques, résultats de campagnes expérimentales réalisées en France ces dernières années (Prezıosa, 1983). Les différences quant à 


\begin{tabular}{|c|c|c|c|c|c|c|c|c|c|c|}
\hline$t_{1}^{-\frac{n}{n}}$ & $\begin{array}{l}8 \\
: \\
7\end{array}$ & $\begin{array}{l}\text { \& } \\
\stackrel{+}{0}\end{array}$ & 悤 & 总 & 文 & 怘 & 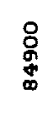 & $\begin{array}{l}\text { o } \\
\text { of } \\
0 \\
0\end{array}$ & $\begin{array}{l}\stackrel{n}{\circ} \\
\stackrel{0}{0} \\
\stackrel{-}{0}\end{array}$ & 京 \\
\hline T粮 & $\begin{array}{l}8 \\
\vdots \\
\vdots \\
0\end{array}$ & $\begin{array}{l}8 \\
\vdots\end{array}$ & $\begin{array}{l}\stackrel{8}{0} \\
\text { o. }\end{array}$ & $\begin{array}{l}\stackrel{\bigcirc}{\vdots} \\
\text { d }\end{array}$ & 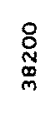 & 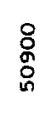 & $\begin{array}{l}\stackrel{\circ}{\circ} \\
\stackrel{a}{a}\end{array}$ & $\begin{array}{l}8 \\
0 \\
0 \\
0 \\
n\end{array}$ & $\begin{array}{l}8 \\
0 \\
0 \\
0 \\
0\end{array}$ & : \\
\hline in & ${ }_{0}^{\infty}$ & $\underset{\$}{\$}$ & $\stackrel{\circ}{\circ}$ & 足 & 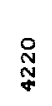 & 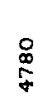 & $\begin{array}{l}\stackrel{8}{\circ} \\
\&\end{array}$ & 虽 & : & $\underset{\exists}{\exists}$ \\
\hline
\end{tabular}

败金空

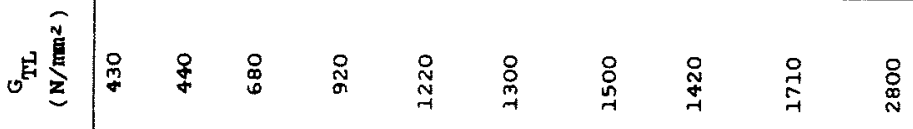

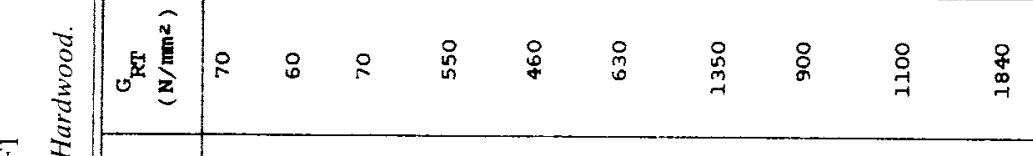

II

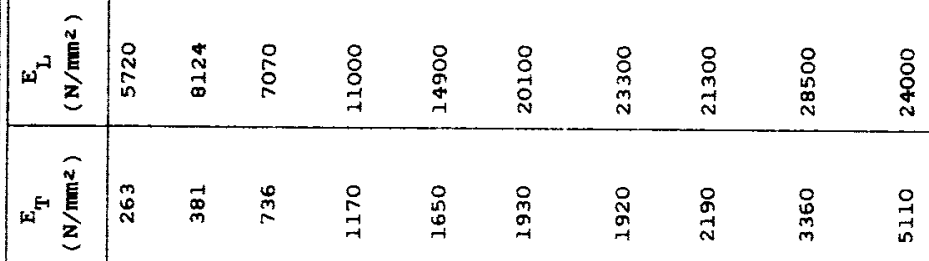

w 䒨

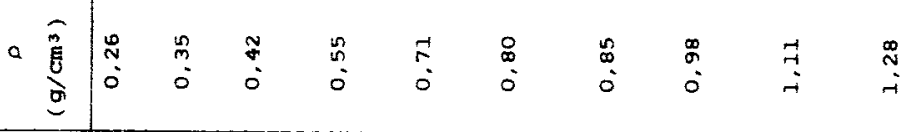

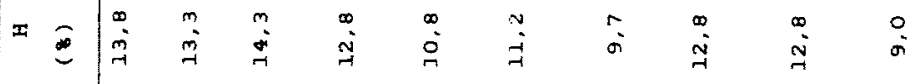

\begin{tabular}{|c|c|c|c|c|c|c|c|c|c|}
\hline 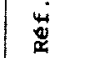 & $\rightarrow$ & $H$ & $\dashv$ & 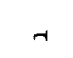 & $\rightarrow$ & $\neg$ & $\leadsto$ & $\leadsto$ & - \\
\hline 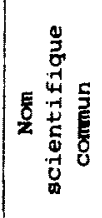 & 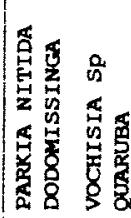 & 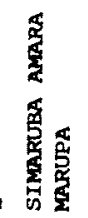 & 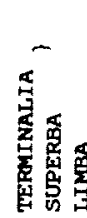 & 8 & - & 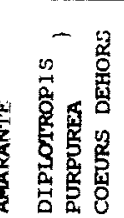 & 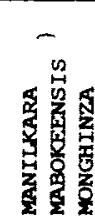 & 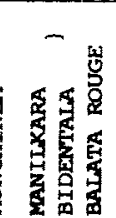 & 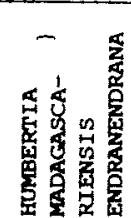 \\
\hline
\end{tabular}




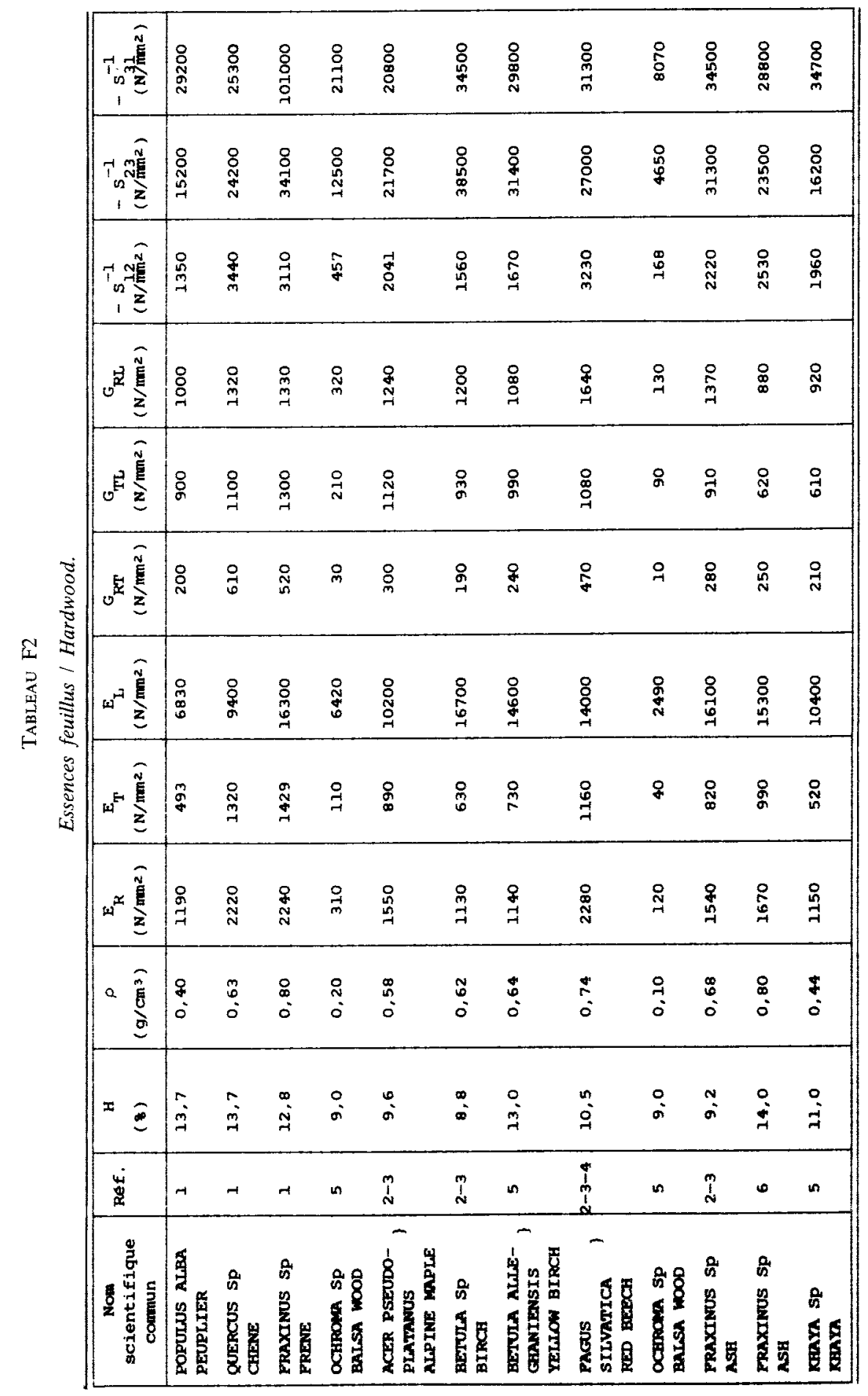




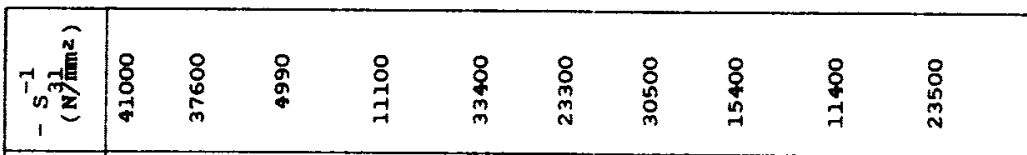

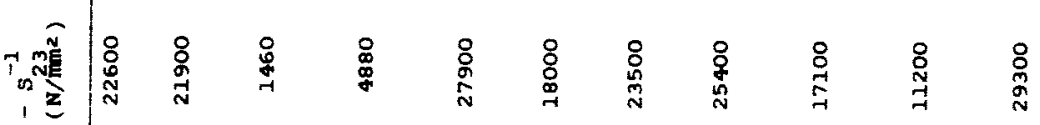

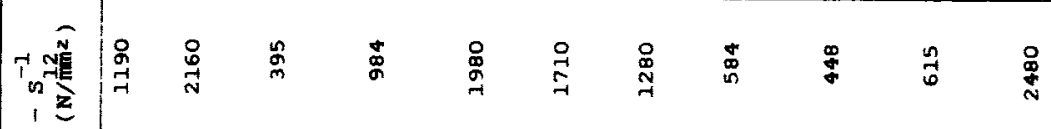

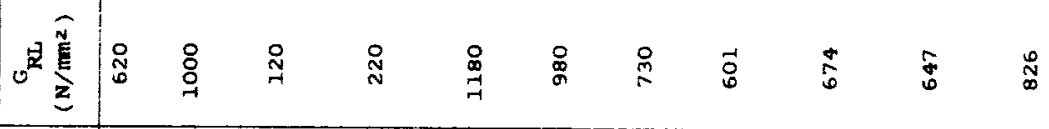

自褰

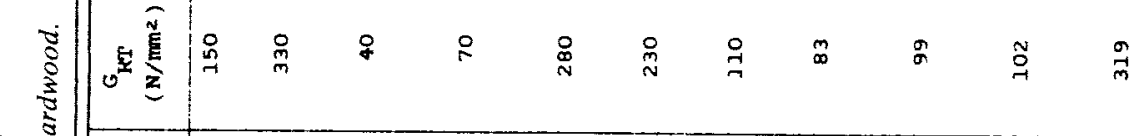

I. $\stackrel{3}{2}$

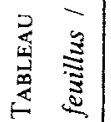

w

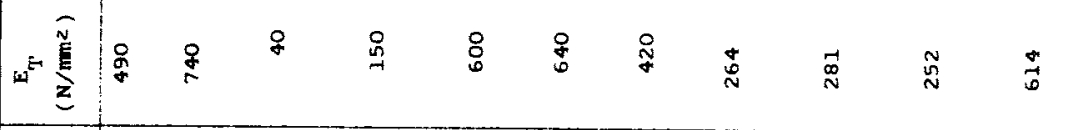

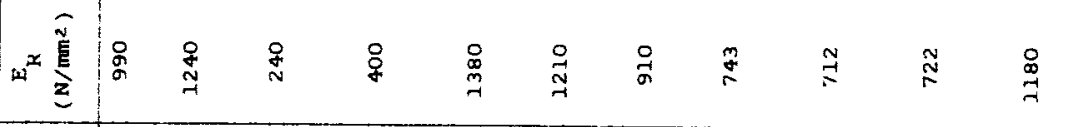

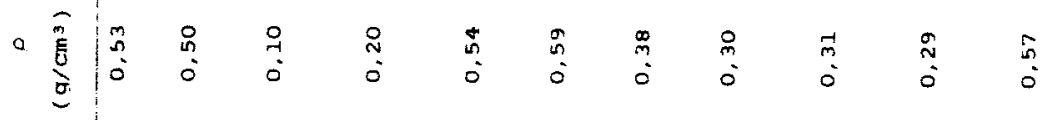

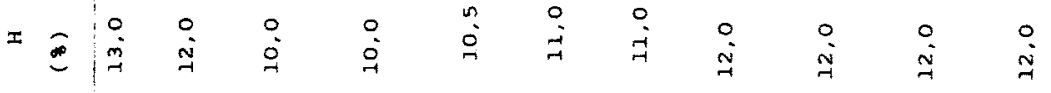

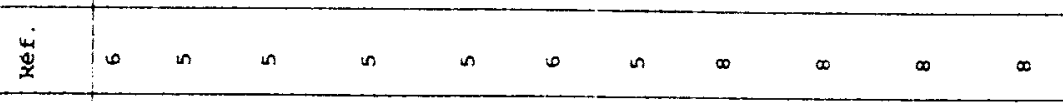

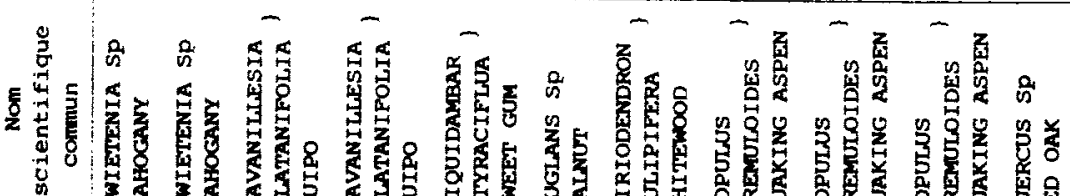
ชิ 


\begin{tabular}{|c|c|c|c|c|c|c|c|c|c|c|}
\hline 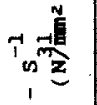 & 怘 & 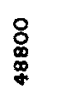 & $\begin{array}{l}: \\
: \\
0 \\
:\end{array}$ & $\begin{array}{l}8 \\
8 \\
0 \\
0\end{array}$ & $\underset{8}{8}$ & $\begin{array}{l}\stackrel{8}{\circ} \\
\stackrel{0}{0} \\
m\end{array}$ & 总 & $\underset{7}{\stackrel{8}{7}}$ & $\frac{8}{8}$ & $\begin{array}{l}\text { : } \\
\text { : }\end{array}$ \\
\hline 1 & \begin{tabular}{l}
8 \\
8 \\
\multirow{2}{N}{} \\
\end{tabular} & 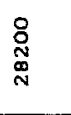 & $\begin{array}{l}8 \\
8 \\
\stackrel{\leftrightarrow}{8}\end{array}$ & $\begin{array}{l}\xi \\
\vdots \\
\sigma\end{array}$ & $\begin{array}{l}8 \\
8 \\
7 \\
7\end{array}$ & $\frac{⿱}{\vdots}$ & 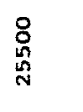 & $\underset{7}{q}$ & $\begin{array}{l}\text { O } \\
\stackrel{D}{\infty} \\
\text { N }\end{array}$ & : \\
\hline$T_{1} \underset{z}{\tilde{N}}$ & $\stackrel{8}{8}$ & $\stackrel{\stackrel{9}{N}}{i}$ & $\stackrel{9}{\stackrel{m}{j}}$ & $\begin{array}{l}0 \\
\stackrel{9}{9} \\
9\end{array}$ & : & $\stackrel{\circ}{\stackrel{D}{d}}$ & $\begin{array}{l}0 \\
\stackrel{8}{0} \\
\stackrel{m}{m}\end{array}$ & $\stackrel{8}{\stackrel{0}{0}}$ & 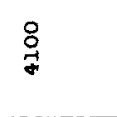 & : \\
\hline$v^{a} \underline{\Sigma}$ & 8 & 杲 & $\stackrel{9}{\stackrel{g}{9}}$ & : & $\underset{7}{\stackrel{2}{7}}$ & 品 & $\stackrel{8}{8}$ & 号 & $\stackrel{9}{\ddagger}$ & 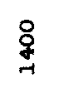 \\
\hline $0^{E}$ & 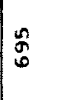 & $\hat{i}$ & $\underset{\infty}{\stackrel{\rho}{\infty}}$ & $\underset{7}{\circ}$ & $\stackrel{\tilde{N}}{\sigma}$ & 品 & $\frac{8}{0}$ & 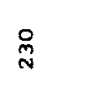 & 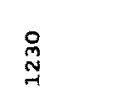 & $\stackrel{9}{\lessgtr}$ \\
\hline 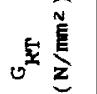 & 怘 & $\vec{\circ}$ & 品 & 吕 & $\stackrel{m}{m}$ & : & Nㅗ & $\underset{\sim}{\stackrel{D}{\infty}}$ & ถి & 只 \\
\hline भit & \begin{tabular}{l}
8 \\
8 \\
$\$$ \\
\hdashline
\end{tabular} & $\underset{\substack{9\\
}}{\stackrel{8}{7}}$ & 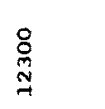 & : & \begin{tabular}{l}
8 \\
8 \\
\hdashline
\end{tabular} & $\underset{7}{7}$ & $\underset{\substack{n\\
}}{\stackrel{8}{7}}$ & $\frac{9}{m}$ & $\underset{8}{8}$ & $\begin{array}{l}\stackrel{8}{0} \\
\stackrel{9}{0}\end{array}$ \\
\hline$=\frac{\widehat{N}}{\underline{E}}$ & 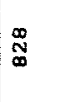 & $\vec{N}$ & 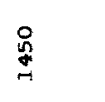 & 怘 & $\stackrel{N}{\approx}$ & $\underset{\infty}{\tilde{D}}$ & $\stackrel{\circ}{\stackrel{7}{7}}$ & 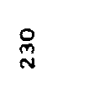 & $\stackrel{\check{n}}{7}$ & : \\
\hline W & $\begin{array}{l}8 \\
0 \\
0 \\
-12\end{array}$ & 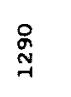 & 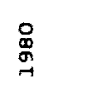 & $\frac{8}{8}$ & $\stackrel{\circ}{\stackrel{P}{m}}$ & ষ⿳亠口冋ౖ & : & 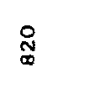 & 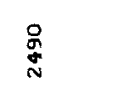 & Nָ \\
\hline$a \frac{\tilde{E}}{E}$ & $\begin{array}{l}0 \\
\vdots \\
0\end{array}$ & $\begin{array}{l}\infty \\
0 \\
0 \\
0\end{array}$ & $\begin{array}{l}\infty \\
0 \\
0\end{array}$ & ¿̊ & $\stackrel{0}{n}$ & $\begin{array}{l}0 \\
6 \\
0 \\
0\end{array}$ & $\stackrel{m}{0}$ & $\stackrel{ \pm}{0}$ & $\stackrel{0}{\circ}$ & $\begin{array}{l}n \\
\infty \\
0\end{array}$ \\
\hline$\pm \hat{\infty}$ & \begin{tabular}{l}
0 \\
\multirow{2}{*}{}
\end{tabular} & $\begin{array}{l}\stackrel{0}{7} \\
\stackrel{7}{*}\end{array}$ & $\stackrel{0}{\dddot{H}}$ & $\stackrel{0}{\stackrel{H}{*}}$ & $\tilde{\sigma}$ & $\frac{\sigma}{\sigma}$ & $\stackrel{0}{\circ}$ & $\dot{0}$ & $\stackrel{0}{\stackrel{9}{4}}$ & $\stackrel{0}{N}$ \\
\hline 这 & $\infty$ & $\infty$ & $\vec{t}$ & $\exists$ & $\stackrel{9}{9}$ & $\stackrel{\circ}{-1}$ & $\stackrel{9}{\circ}$ & $\exists$ & $\overrightarrow{7}$ & $\exists$ \\
\hline 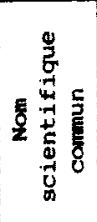 & 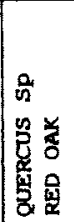 & 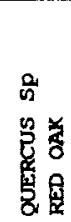 & 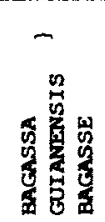 & 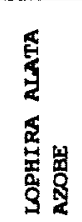 & 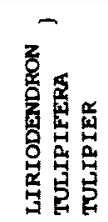 & 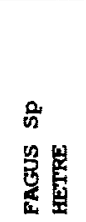 & 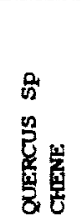 & 一 & 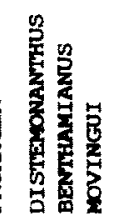 & $\begin{array}{l}5 \\
5 \\
6 \\
6 \\
5 \\
5 \\
5 \\
5\end{array}$ \\
\hline
\end{tabular}




\begin{tabular}{|c|c|c|c|c|c|c|c|c|c|c|c|c|c|}
\hline & 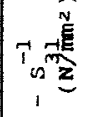 & 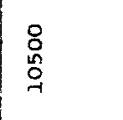 & $\begin{array}{l}8 \\
\stackrel{8}{0} \\
N \\
N\end{array}$ & 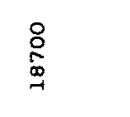 & $\underset{\sim}{\stackrel{3}{*}}$ & $\begin{array}{l}8 \\
\stackrel{0}{7} \\
7\end{array}$ & $\frac{9}{9}$ & 量 & $\begin{array}{l}\text { : } \\
\stackrel{9}{\$} \\
\stackrel{-}{-1}\end{array}$ & 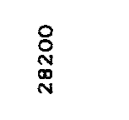 & $\begin{array}{l}8 \\
\stackrel{8}{7} \\
m\end{array}$ & $\begin{array}{l}\stackrel{\Xi}{N} \\
\text { N }\end{array}$ & $\begin{array}{l}\frac{8}{8} \\
\frac{8}{8}\end{array}$ \\
\hline & 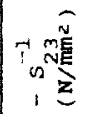 & 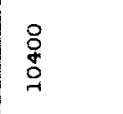 & 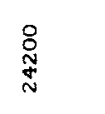 & $\begin{array}{l}\stackrel{8}{\$} \\
\text { \& }\end{array}$ & $\begin{array}{l}\stackrel{M}{\mathcal{H}} \\
\stackrel{D}{0} \\
\stackrel{-}{0}\end{array}$ & \begin{tabular}{l}
8 \\
$\vdots$ \\
\hdashline
\end{tabular} & $\begin{array}{l}\text { 呆 } \\
\text { i } \\
\text { in }\end{array}$ & $\underset{\substack{8 \\
\hdashline}}{8}$ & $\begin{array}{l}\stackrel{0}{0} \\
\text { o } \\
\text { न }\end{array}$ & $\stackrel{8}{\stackrel{8}{N}}$ & $\begin{array}{l}\stackrel{8}{0} \\
\stackrel{9}{0}\end{array}$ & $\begin{array}{l}8 \\
\stackrel{8}{9} \\
\stackrel{-1}{-1}\end{array}$ & $\underset{8}{\stackrel{8}{8}}$ \\
\hline \multirow{11}{*}{ 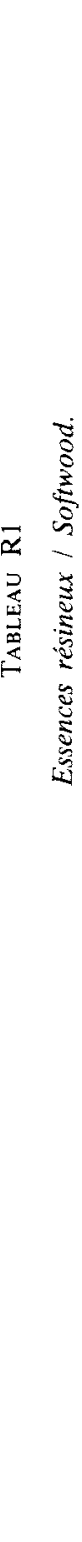 } & $\begin{array}{r}+N \tilde{N} \\
n^{n}=\frac{\tilde{E}}{2} \\
1\end{array}$ & $\begin{array}{l}\stackrel{\circ}{\circ} \\
\text { \& } \\
\text { - }\end{array}$ & $\underset{\sim}{\stackrel{N}{\sim}}$ & 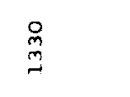 & $\stackrel{m}{2}$ & $\underset{0}{0}$ & 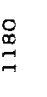 & $\begin{array}{l}8 \\
8 \\
8\end{array}$ & 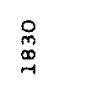 & $\stackrel{i}{i}$ & $\stackrel{8}{\stackrel{0}{7}}$ & 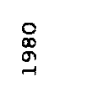 & 胥 \\
\hline & 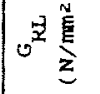 & $\stackrel{0}{\stackrel{2}{2}}$ & 总 & $\frac{5}{4}$ & $\stackrel{+}{\infty}$ & 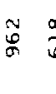 & $\begin{array}{l}\infty \\
\text {-1 } \\
0\end{array}$ & 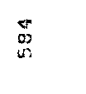 & $\stackrel{\infty}{\infty}$ & $\stackrel{\infty}{\sim}$ & $\stackrel{m}{i}$ & : & స్ \\
\hline & $0^{e} \frac{\tilde{E}}{\Sigma}$ & 3 & $\vec{q}$ & $\stackrel{\tilde{N}}{\mathfrak{O}}$ & 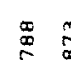 & $\frac{n}{\infty} \stackrel{4}{C}$ & $\stackrel{2}{2}$ & 8 & 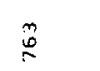 & $\underset{\xi}{\xi}$ & $\stackrel{\substack{\varpi \\
\varpi}}{*}$ & $\bar{\Phi}$ & $\vec{D}$ \\
\hline & $0^{5} \frac{\tilde{E}}{\underline{E}}$ & $\vec{n}$ & $\mathfrak{q}$ & $\Phi$ & $\vec{r}$ & 2 & ถิ & $\underset{n \rightarrow}{x}$ & $\underset{\curvearrowright}{\infty}$ & 5 & $\overrightarrow{5}$ & $\Rightarrow$ & $\tilde{*}$ \\
\hline & $=\stackrel{\substack{\tilde{E} \\
z}}{\Sigma}$ & $\begin{array}{c}q \\
\substack{0 \\
m \\
m}\end{array}$ & : & 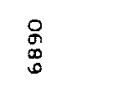 & $\begin{array}{ll}N & 0 \\
0 & 0 \\
0 & 0\end{array}$ & $\begin{array}{ll}0 & 0 \\
: & 0 \\
0 & 0 \\
0 & 0\end{array}$ & 号 & $\begin{array}{l}a \\
a \\
a \\
b \\
b\end{array}$ & $\frac{q}{5}$ & $\stackrel{\circ}{\stackrel{2}{\alpha}}$ & $\underset{\stackrel{8}{0}}{\stackrel{0}{0}}$ & $\stackrel{\stackrel{0}{n}}{=}$ & 禺 \\
\hline & $x=\frac{\tilde{\tilde{E}}}{\underline{z}}$ & $\hat{\Phi}$ & $\stackrel{+}{\circ}$ & $\stackrel{+}{\dot{m}}$ & $\vec{D} \stackrel{\vec{n}}{\vec{n}}$ & $\stackrel{\substack{n \\
m}}{\stackrel{n}{n}}$ & $\frac{\pi}{n}$ & 岕: & 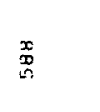 & 点 & $\stackrel{\infty}{\stackrel{\infty}{*}}$ & $\frac{8}{2}$ & $\tilde{j}$ \\
\hline & wex $\underset{\Sigma}{\frac{v}{z}}$ & $\begin{array}{l}q \\
\vdots \\
0\end{array}$ & $\stackrel{0}{\infty}$ & $\stackrel{m}{\stackrel{m}{p}}$ & 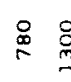 & 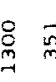 & $\vec{n}$ & id & 离 & $\stackrel{\infty}{\stackrel{\infty}{N}}$ & $\stackrel{a}{6}$ & 品 & $\stackrel{8}{8}$ \\
\hline & ○ & $\begin{array}{l}\stackrel{0}{0} \\
\vdots\end{array}$ & $\vec{m}$ & $\ddot{m}$ & 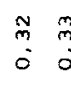 & $\begin{array}{ll}m & \tilde{m} \\
\dot{m} & 0\end{array}$ & ? & $\begin{array}{l}x \\
N \\
0\end{array}$ & $\stackrel{0}{0}_{0}^{\circ}$ & $\stackrel{4}{\stackrel{4}{0}}$ & $\stackrel{0}{m}$ & $\vec{\ddagger}$ & $\stackrel{+}{\stackrel{+}{0}}$ \\
\hline & $x$ & $\begin{array}{l}N \\
- \\
-\end{array}$ & $\vec{m}$ & $\stackrel{\sim}{\stackrel{N}{\sim}}$ & 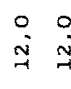 & 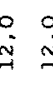 & \begin{tabular}{l}
0 \\
\multirow{N}{N}{}
\end{tabular} & $\dddot{m}$ & 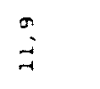 & g & $\stackrel{\infty}{\sim}$ & $\stackrel{q}{i}$ & $\stackrel{3}{3}$ \\
\hline & 峞 & $\infty$ & $\infty$ & $\infty$ & $\infty \infty$ & $\infty \infty$ & $\infty$ & $\infty$ & $\infty$ & $\infty$ & $\infty$ & $\mathbf{\infty}$ & $\infty$ \\
\hline & 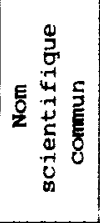 & 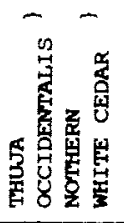 & 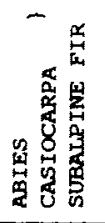 & 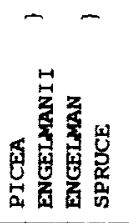 & & $=$ & 5 & 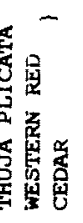 & 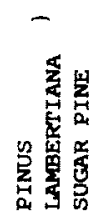 & 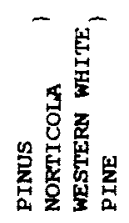 & 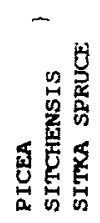 & 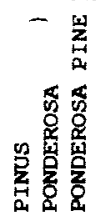 & 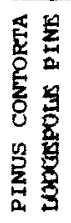 \\
\hline
\end{tabular}




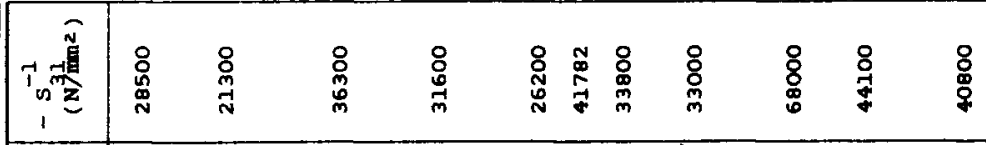

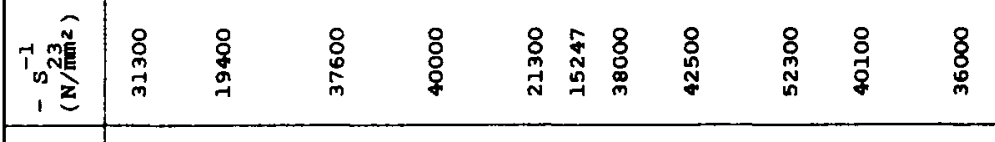

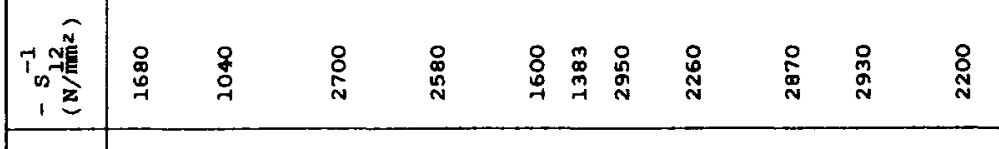

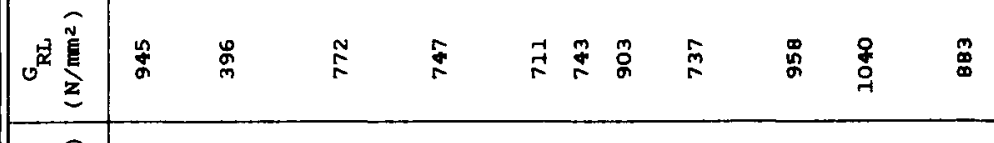

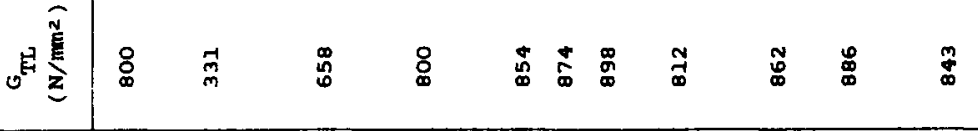

0

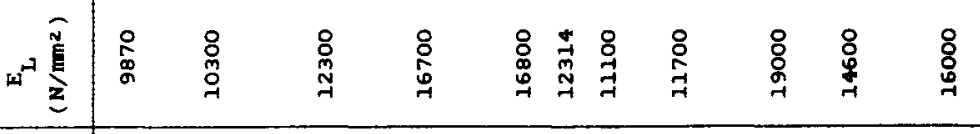

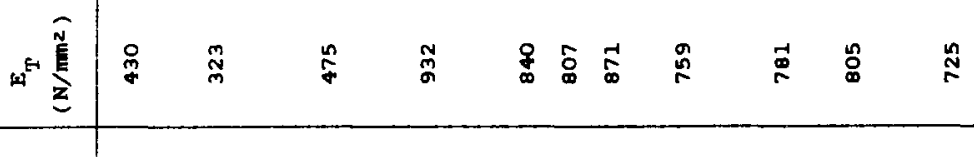

బ=

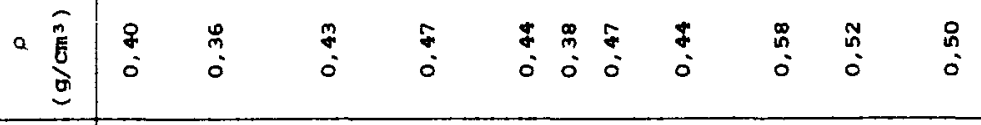

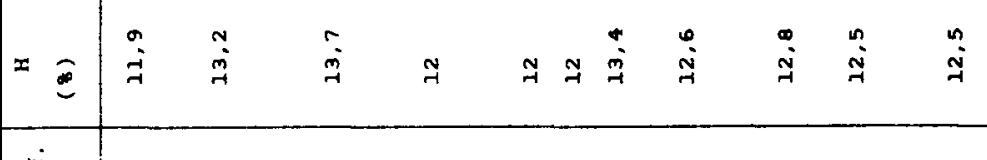

\begin{tabular}{l|lllllllllll}
\hline & $\infty$ & $\infty$ & $\infty$ & $\infty$ & $\infty$ & $\infty$ & $\infty$ & $\infty$ & $\infty$ & $\infty$ & $\infty$
\end{tabular}

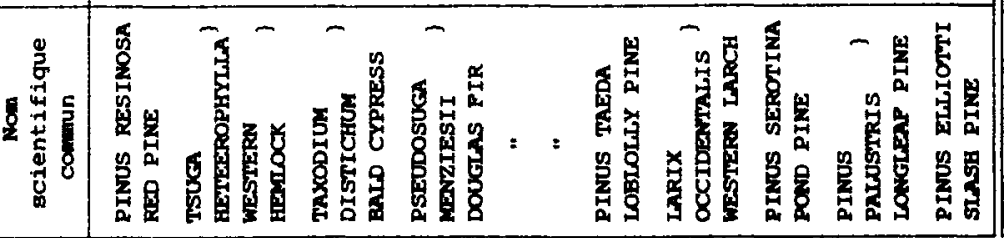




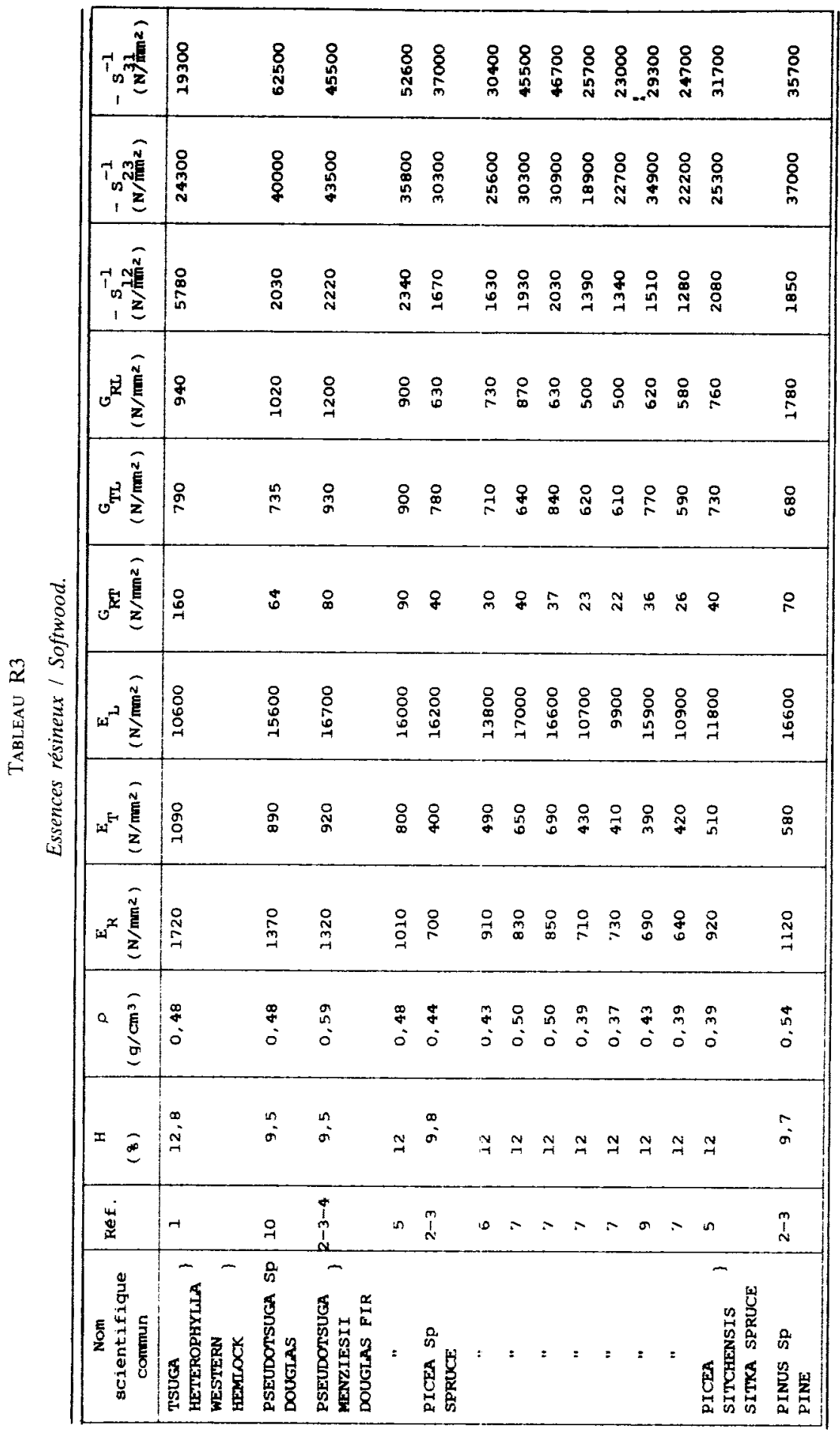


l'interprétation des données expérimentales correspondant aux termes non diagonaux de la matrice d'élasticité, ont pour conséquence une meilleure cohérence interne des modèles prévisionnels proposés.

\section{Les constantes élastiques : définitions, notations}

Le matériau bois est considéré comme milieu continu, élastique, macroscopiquement homogène à symétrie matérielle orthotrope cylindrique (GuITaRD, 1985) d'axes principaux suivant les directions radiale (R), tangentielle (T) et Iongitudinale (L) auxquelles correspondent les coordonnées spatiales cylindriques $(r, \theta, z)$, comptées à partir de l'axe de la grume.

Rappelons, pour des considérations de clarté de notations, que dans ce système d'axes, la loi de comportement élastique permet d'expliciter par exemple, les composantes $\epsilon_{i j}$ du tenseur des déformations en fonction des composantes $\sigma_{i j}$ du tenseur des contraintes à l'aide de neuf constantes élastiques linéairement indépendantes.

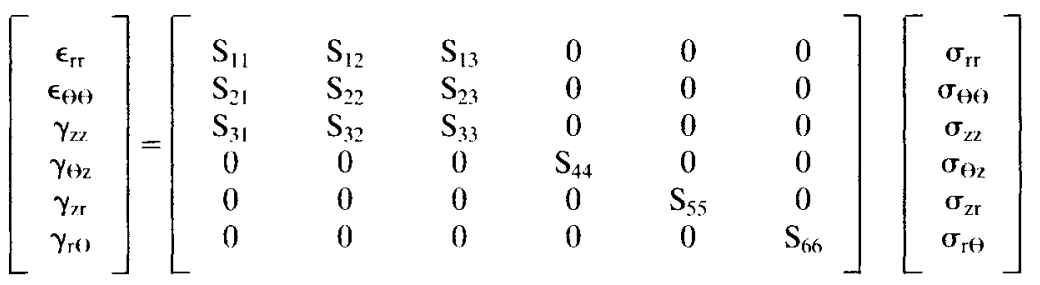

La matrice des complaisances élastiques, transcrite ici avec une contraction d'indices conventionnelle (Guitard, 1987), est une matrice en théorie symétrique. Cette propriété résulte nécessairement de l'hypothèse d'existence d'un potentiel élastique forme quadratique définie positive des composantes des tenseurs des contraintes.

Par tradition, l'ingénieur utilise 12 grandeurs techniques pour caractériser le comportement élastique du bois, qui sont liées aux neuf complaisances élastiques par les relations suivantes :

- Trois modules d'Young :

$$
\mathrm{E}_{\mathrm{R}}=\mathrm{S}_{11}^{-1} ; \mathrm{E}_{\mathrm{T}}=\mathrm{S}_{22}^{-1} ; \mathrm{E}_{\mathrm{L}}=\mathrm{S}_{3,3}^{-1}
$$

- Trois modules de cisaillement :

$$
\mathrm{G}_{\mathrm{TI}}=\mathrm{S}_{44}^{-1} ; \mathrm{G}_{\mathrm{LR}}=\mathrm{S}_{55}^{-1} ; \mathrm{G}_{\mathrm{RT}}=\mathrm{S}_{\mathrm{6 \sigma}}^{-1}
$$

- Six coefficients de Poisson :

$$
v_{\mathrm{RT}}, v_{\mathrm{TR}}, v_{\mathrm{TL}}, v_{\mathrm{LT}}, v_{\mathrm{LR}}, v_{\mathrm{R}} \text {. }
$$

qui satisfont aux relations de symétrie suivantes :

$$
\begin{aligned}
& \mathrm{S}_{\mathrm{L} 2}=\mathrm{S}_{21}=-\frac{\nu_{\mathrm{RT}}}{\mathrm{E}_{\mathrm{R}}}=-\frac{\nu_{\mathrm{TR}}}{\mathrm{E}_{\mathrm{T}}} ; \mathrm{S}_{23}=\mathrm{S}_{32}=-\frac{\nu_{\mathrm{TL}}}{\mathrm{E}_{\mathrm{T}}}=-\frac{\nu_{\mathrm{LT}}}{\mathrm{E}_{\mathrm{L}}} ; \\
& \mathrm{S}_{31}=\mathrm{S}_{13}=-\frac{\nu_{\mathrm{L} . \mathrm{R}}}{\mathrm{E}_{\mathrm{L}}}=-\frac{\nu_{\mathrm{RL}}}{\mathrm{E}_{\mathrm{R}}}
\end{aligned}
$$




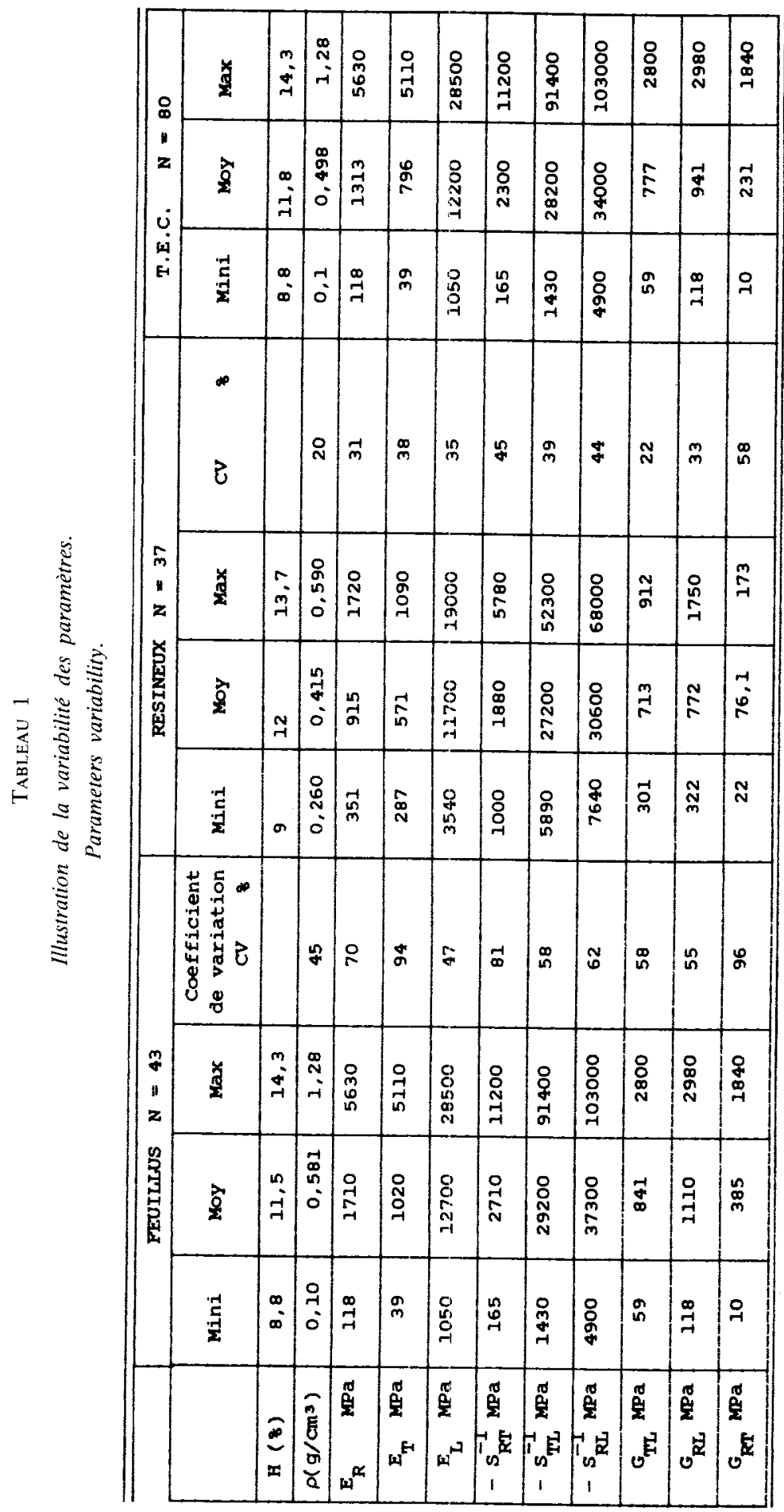




\section{Matériels : constitution de la banque de données}

Les tableaux récapitulatifs reproduits en annexe, contiennent les éléments actuellement pris en compte dans la banque de données informatisée, disponible sur disquette et exploitable sur un système Apple II E.

Les références bibliographiques mentionnées dans ces tableaux ont été exploitées avec un soin particulier qui a permis d'éliminer les redondances possibles dans les publications successives.

Pour chaque individu (bois) répertorié, les paramètres retenus sont :

$\mathrm{H}$ : taux d'humidité en $\%$ dans les conditions d'essai.

$\rho$ : masse volumique en $\mathrm{g} / \mathrm{cm}^{3}$ à l'humidité $\mathrm{H}$ d'essai.

$\mathrm{S}_{\mathrm{ij}}$ : neuf complaisances élastiques, correspondant aux définitions ci-dessus.

Remarque : De nombreux auteurs présentent leurs résultats en faisant apparaître les grandeurs techniques élastiques, nous les avons exprimées sous forme de complaisances élastiques :

Les termes non diagonaux de la matrice des complaisances sont alors calculés de la façon suivante :

- Lorsque l'auteur indique les coefficients de Poisson nécessaires :

$$
\mathrm{S}_{\mathrm{ij}}=\mathrm{S}_{\mathrm{ji}}=-\frac{1}{2}\left(\frac{v_{\mathrm{ij}}}{\mathrm{E}_{\mathrm{i}}}+\frac{\nu_{\mathrm{ji}}}{\mathrm{E}_{\mathrm{j}}}\right) \text { pour } \mathrm{i} \neq \mathrm{j}
$$

- Lorque l'auteur n’indique que l'un des coefficients de Poisson nécessaires :

$$
S_{i j}=S_{j i}=-\frac{\nu_{i j}}{E_{i}}
$$

Cette démarche a pour conséquence de symétriser les matrices d'élasticité prises en compte, alors que les résultats expérimentaux ne vérifient pas nécessairement cette condition théorique.

La bibliothèque comprend, toutes essences confondues, un effectif total $\mathrm{N}=80$ individus, qualifiés chacun par deux paramètres physiques et neuf caractères élastiques. Cette population totale comprend deux sous populations complémentaires, une population notée « $F$ » de bois feuillus à effectif de 43 individus, et une population notée « $\mathrm{R}$ » de bois résineux à effectif de 37 individus. Ces individus appartiennent ou non à des espèces différentes. Un individu peut être la moyenne de certain nombre de points expérimentaux.

\section{Traitements statistiques}

Les traitements statistiques mis en ceuvre permettent de rechercher les régressions simples ( 1 paramètre explicatif $\mathrm{X}$ ) ou multiples ( 2 paramètres explicatifs $\mathrm{X}$ et $\mathrm{Y}$ ) de la variable $Z$, sous forme de lois :

$$
\begin{gathered}
\text { linéaires: } \mathrm{Z}=\mathrm{A}+\mathrm{B}_{1} \mathrm{X}+\mathrm{B}_{2} \mathrm{Y} \\
\text { puissances : } \mathrm{Z}=\mathrm{A} X^{\mathrm{B} 1} \mathrm{Y}^{\mathrm{B} 2}
\end{gathered}
$$




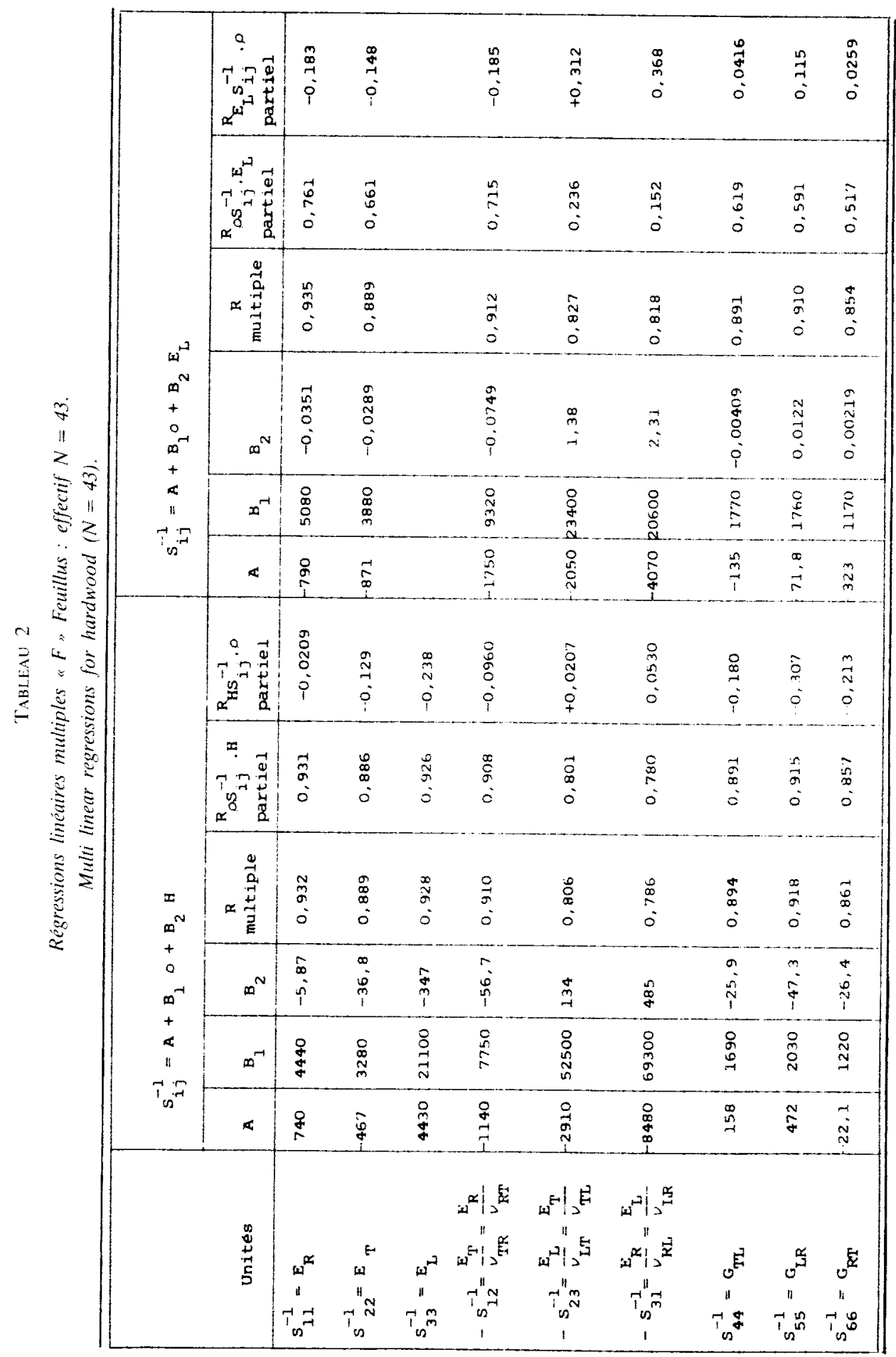


La pertinence des régressions simples, est appréciée par le calcul d'un coefficient de corrélation $R_{z x}$ défini par :

$$
\mathrm{R}_{\mathrm{zx}}=\sqrt{1-\frac{\text { [variance résiduelle] }}{\text { [variance totale] }}}
$$

Dans ces conditions, qu'il s'agisse d'une loi linéaire, ou d'une loi puissance, les coefficients calculés sont une mesure, définie positive, de la dispersion du nuage de points étudiés, autour de la courbe de régression choisie dans une même représentation graphique.

La qualité des régressions linéaires multiples est qualifiée de façon classique (NePveu, 1983) par la donnée du coefficient de corrélation multiple $R_{z}(x, y)$ et des coefficients de corrélation partiels $R_{x z \cdot y}$ et $R_{y z \cdot x}$. Enfin, la variabilité d'un paramètre $X$ est qualifiée par un coefficient de variation $\mathrm{CV}$ exprimé en \% :

$$
\mathrm{CV} \%=\frac{\sqrt{\text { variance totale }}}{\text { valeur moyenne }} \times 100
$$

La variabilité résiduelle d'une variable $Z$, expliquée par un paramètre $X$, est qualifiée par un coefficient de variation résiduelle noté CVR exprimé en \%.

$$
\text { CVR } \%=\frac{\sqrt{\text { variance résiduelle }}}{\text { valeur moyenne }} \times 100
$$

\section{Résultats}

La recherche des corrélations entre les 11 caractères répertoriés par individu, et la présentation des résultats, résultent d'une démarche guidée par la remarque fondamentale suivante :

La caractéristique élastique de première nécessité technologique est le module d'Young $E_{L}$, mesuré suivant la direction du fil d'un bois de masse volumique $\rho$, au taux d'humidité $H$.

Le tableau 1 illustre la variabilité des caractéristiques des bois en précisant pour chacun des paramètres: les valeurs minimale, moyenne et maximale, ainsi que les coefficients de variation $\mathrm{CV} \%$.

Il est fondamental de noter que les bornes maxi et mini, observées pour la population «T.E.C. toutes essences confondues», sont strictement celles de la sous population « $\mathrm{F}$ » des feuillus, laquelle couvre la plus large plage de masses volumiques $\rho\left(0,10\right.$ à $\left.1,28 \mathrm{~g} / \mathrm{cm}^{3}\right)$.

Pour les données disponibles, la variabilité totale des propriétés physiques et mécaniques des résineux, population " $R$ ", est incluse dans celle des " $F$ » et considérablement plus réduite (masses volumiques comprises entre 0,26 et $0,59 \mathrm{~g} / \mathrm{cm}^{3}$ ).

En conséquence, les commentaires porteront dans la suite de cet article sur les deux sous-populations « $R$ » et « $F$ », une analyse plus complète sera présentée dans un mémoire de thèse ( $E_{L}$ AMRI, à paraître). 


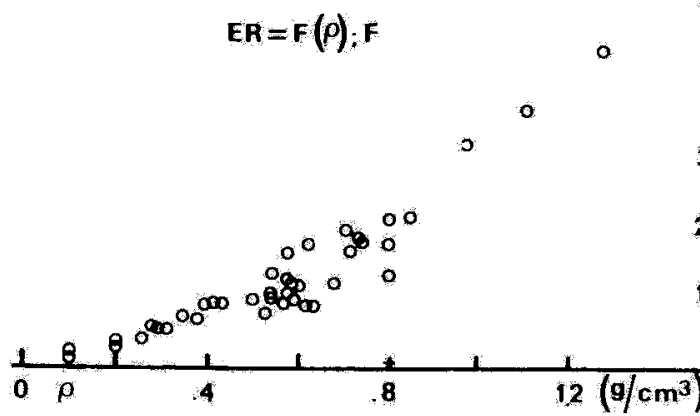

Fic. 1

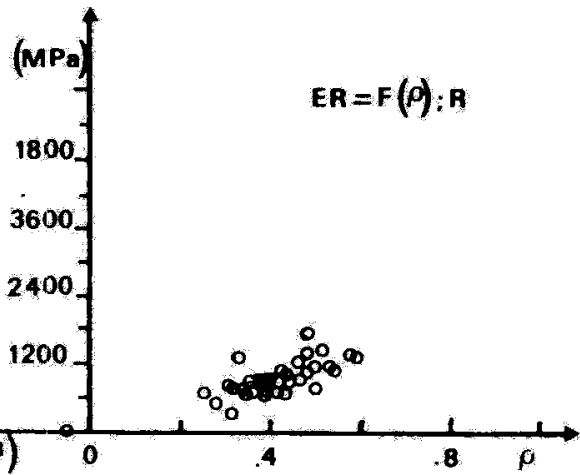

FIG. 10

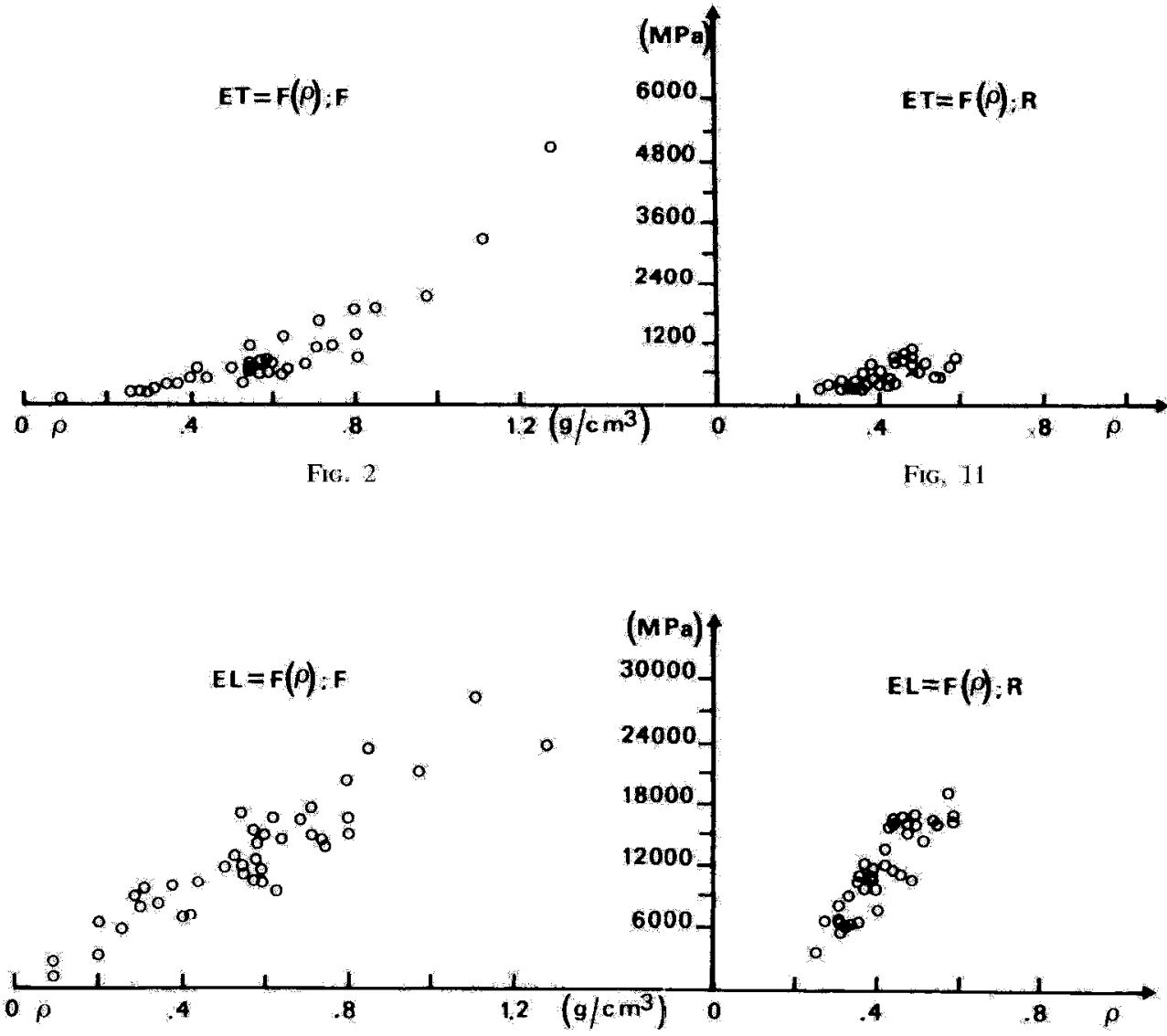

F16. 3

Fic, 12 


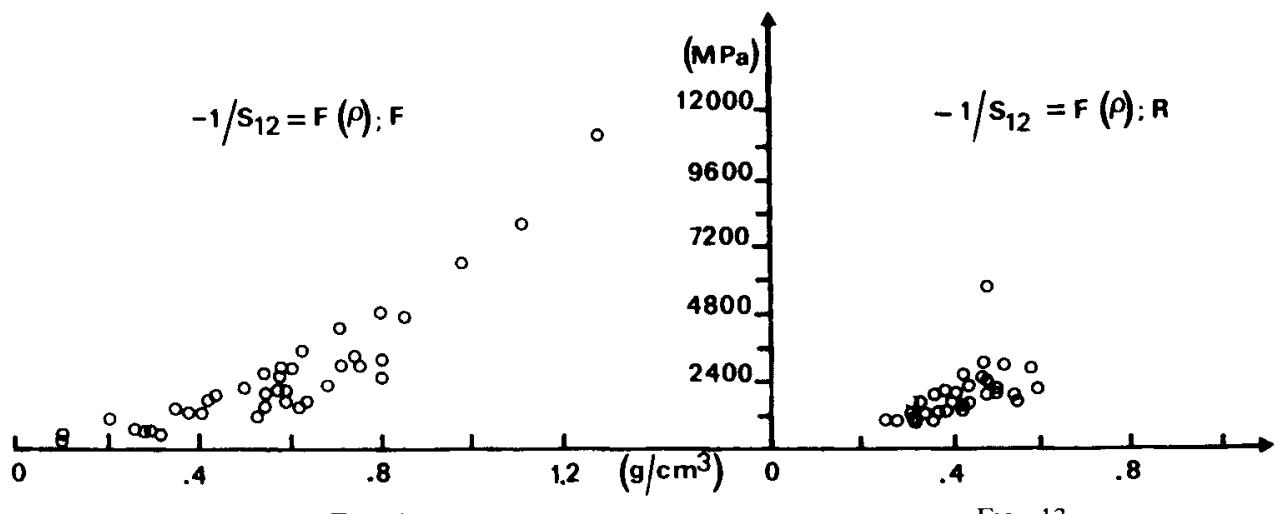

FIG. 4

Fig. 13

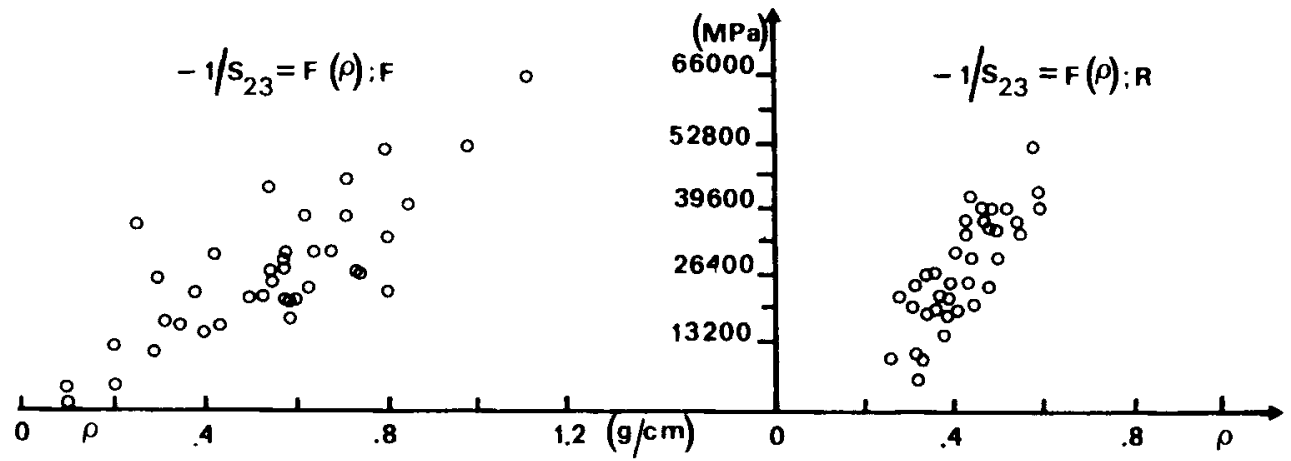

FIG. 5

FIG. 14

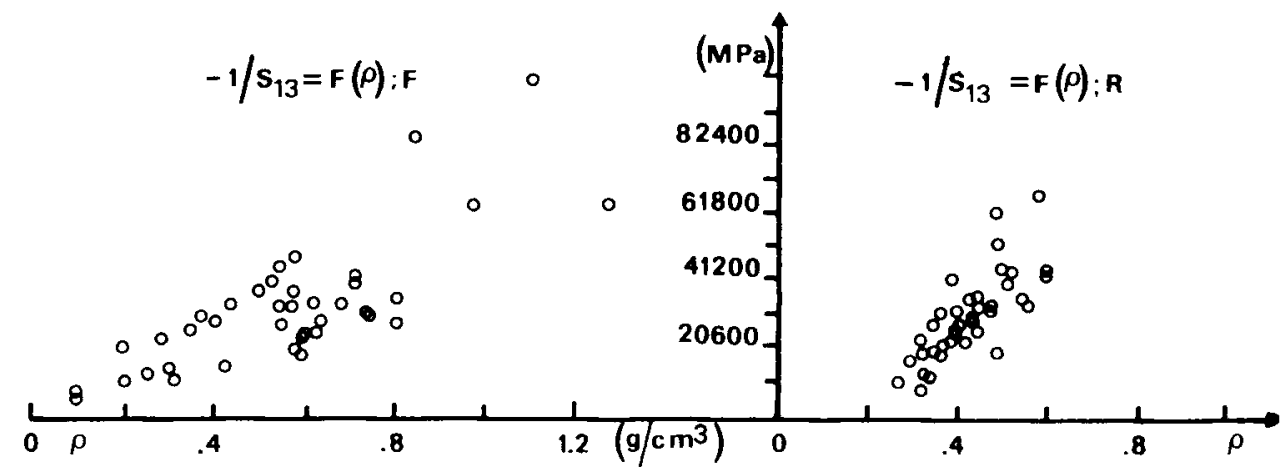

FIG. 6

Fig. 15 


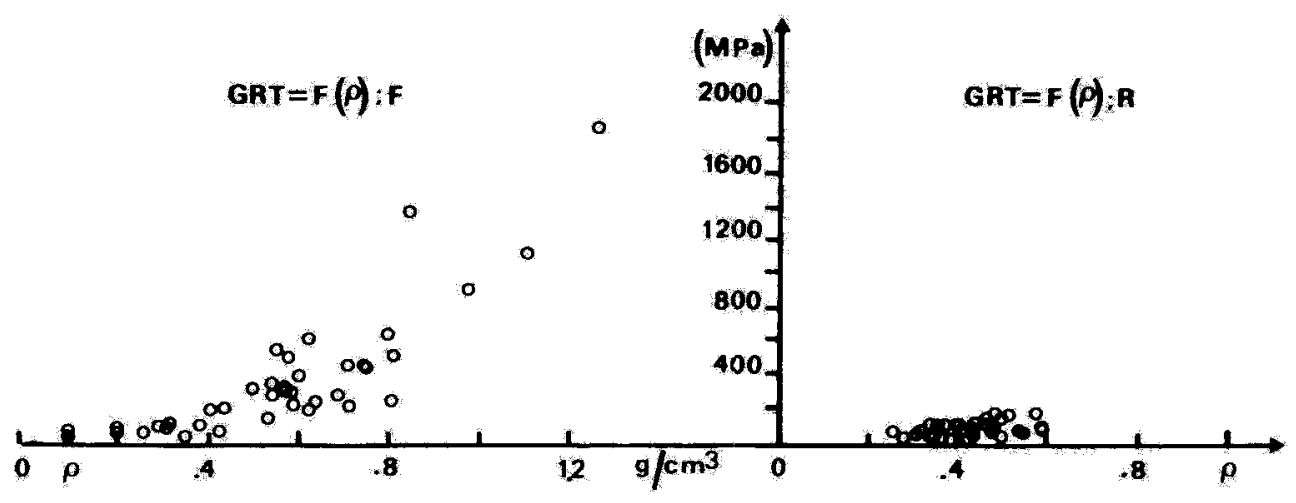

Fig. 7

Fig. 16

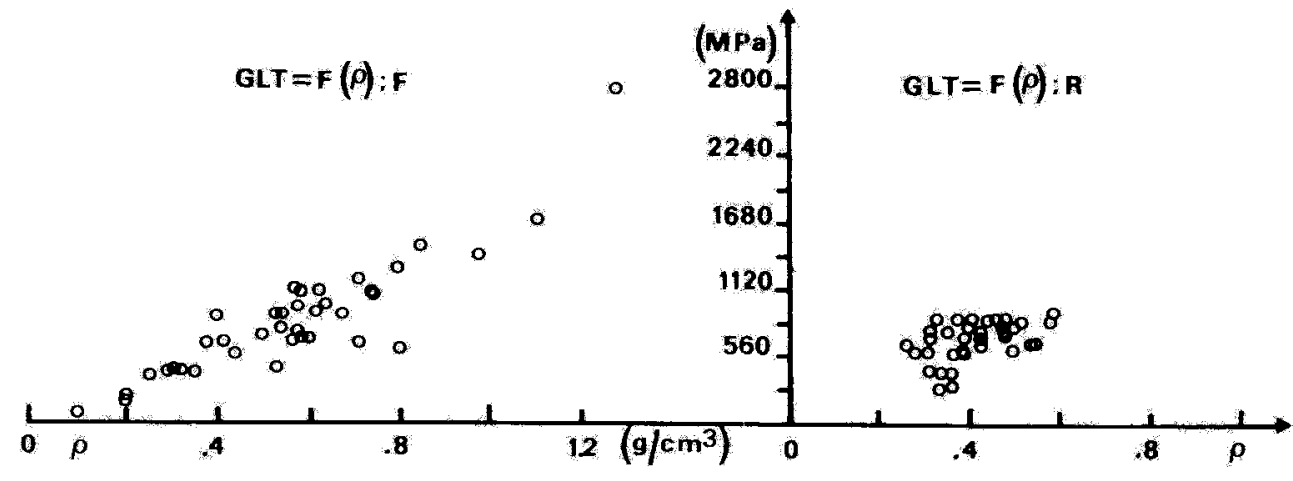

Fic. 8

Fig: 17

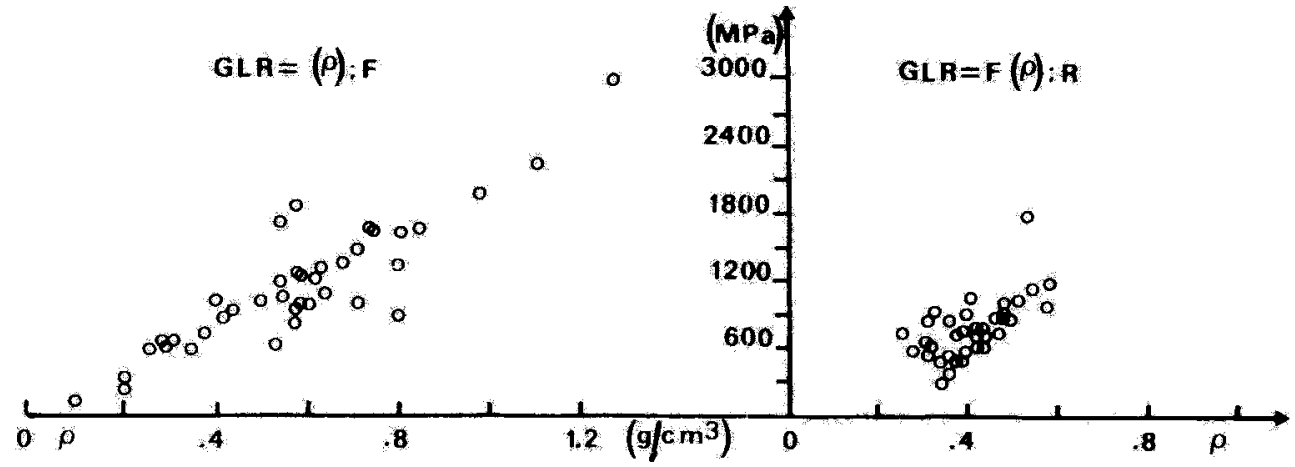

FIG. 9

Fig. 18 


\subsection{Un modèle prévisionnel de comportement élastique tridimensionnel pour les "bois feuillus"}

Le tableau 2 donne les caractéristiques élastiques de bois feuillus sous forme de régressions linéaires multiples à deux paramètres explicatifs, soit, les couples $(\rho, H)$, et $\left(\rho, E_{L}\right)$.

Dans les deux cas, les coefficients de corrélations multiples sont très significatifs, supérieurs à $\mathbf{R}=0,4843$ valeur limite au seuil de $1 \%$ pour un nombre de degrés de liberté ici égal à $\mathrm{N}-2=41$.

L'examen des régressions linéaires multiples donnant les caractéristiques élastiques en fonction des deux paramètres physiques, masse volumique $\rho$ et taux d'humidité $H$, conduit aux commentaires suivants :

La masse volumique $\rho$ est la variable physique explicative majeure de la variabilité totale des constantes élastiques. Les pourcentages de la variance totale expliquée par $\rho$ pour les différentes constantes élastiques sont les suivantes:

\begin{tabular}{|c|c|c|c|c|c|c|c|c|c|}
\hline | Complaisances $^{-1} \mid$ & $S_{11}^{\prime}$ & $\mathrm{S}_{22}^{-1}$ & $S_{33}^{-1}$ & $-S_{12}^{-1}$ & $-S_{23}^{-1}$ & $-S_{31}^{-1}$ & $S_{44}^{-1}$ & $\mathrm{~S}_{55}^{-1}$ & $S_{t h t}$ \\
\hline $\mathrm{R}_{z X}^{2} \times 100$ & 87 & 78 & 86 & 82 & 64 & 61 & 79 & 71 & 73 \\
\hline
\end{tabular}

Le taux d'humidité $\mathrm{H}$ donne des coefficients de corrélation partiels qui révèlent les tendances globales dans le sens prévisible (Gerhards, 1982), à savoir, une diminution des rigidités avec accroissement du taux d'humidité. Toutefois les coefficients sont non significatifs. La banque de données ne fait en effet intervenir que des mesures effectuées sur des bois stabilisés à des taux d'humidité, compris dans une fourchette étroite ( 9 à 14 p. 100) et le plus souvent voisins de 11,5 p. 100. L'influence de ce paramètre $H$ ne sera plus discutée dans ce qui suit.

La seconde partie du tableau 2 permet d'examiner la pertinence de la donnéc d'une caractéristique physique $\rho$ et d'une caractéristique élastique $E_{1}$ pour prévoir les autres caractéristiques élastiques.

La forte corrélation de $E_{L}$ à $\rho$ observée ci-dessus, est bien évidemment confirmée ici, $\rho$ reste en effet la variable explicative majeure, exception faite pour les deux composantes non diagonales $S_{23}$ et $S_{31}$ qui sont mieux expliquées par le couple $\left(\rho, E_{L}\right)$.

Sur les figures 1 à 9 , les points représentatifs de chaque caractéristique élastique sont portés en fonction de la masse volumique. L'examen de ces nuages de points. suggère de discuter l'utilisation de lois de régressions en puissance de $\rho$. Dans ce but, le tableau 3 indique les régressions simples, linéaire et puissance de $\rho$ correspondant à chaque constante élastique, exprimées en prenant comme masse volumique de référence $\bar{\rho}=0,65 \mathrm{~g} / \mathrm{cm}^{3}$, valeur acceptable pour les bois feuillus employés industriellement.

La discrimination entre la loi linéaire et la loi puissance ne peut se dégager simplement d'une comparaison des coefficients de corrélations qui restent très voisins dans tous les cas. 


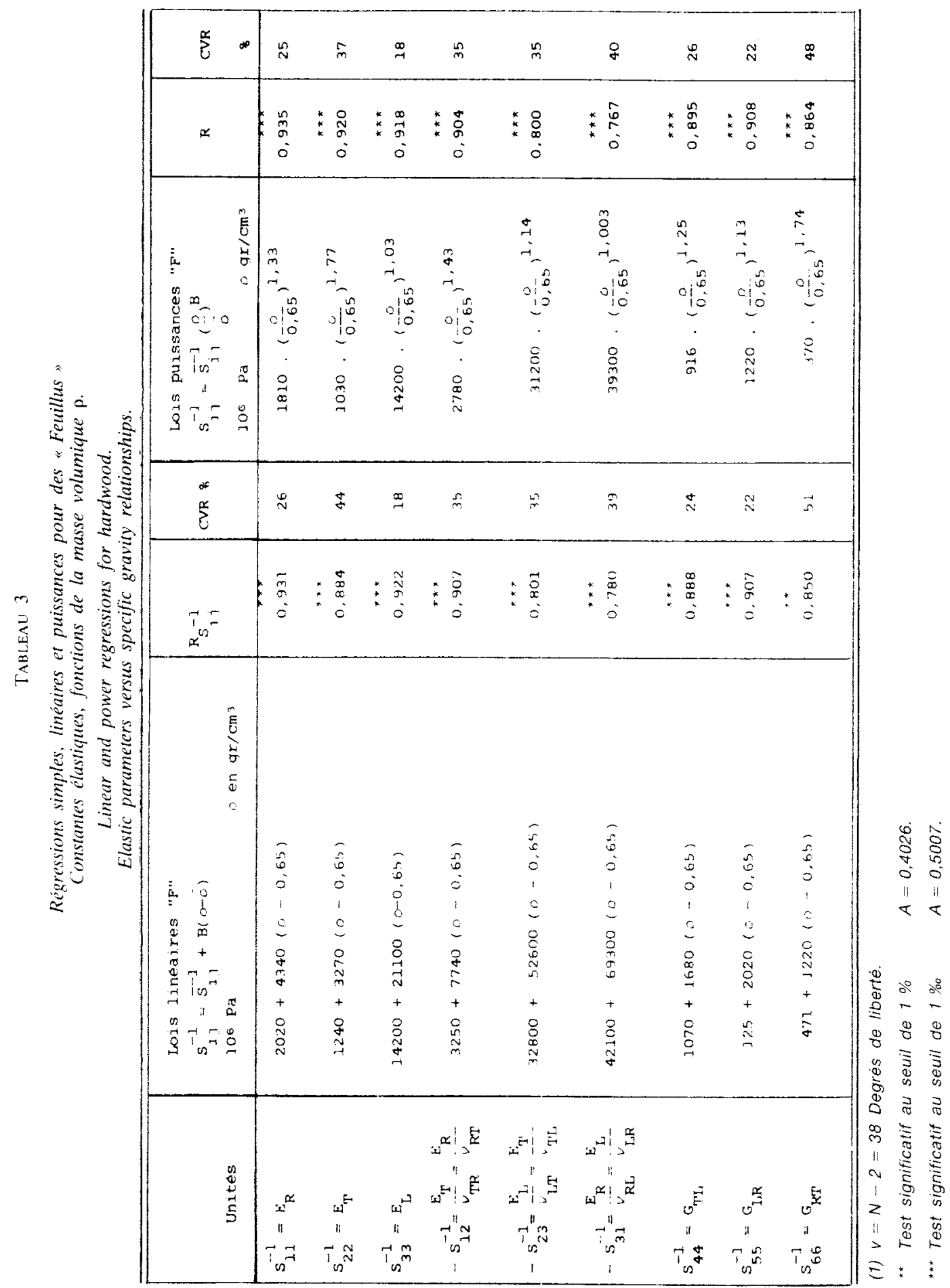


Les régressions linéaires sont biaisées, elles conduisent en effet à des prévisions de rigidités négatives, pour des valeurs faibles, mais interpolées de $\rho$. En conséquence, le modèle prévisionnel de comportement élastique retenu est donné par les lois puissances du tableau 3. Toutes les grandeurs dans ce tableau sont indiquées par des nombres arrondis à 3 chiffres significatifs.

\subsection{Un modèle prévisionnel de comportement élastique tridimensionnel pour les bois résineux}

La population « $\mathrm{R}$ » bois résineux est caractérisée par une plage de masse volumique sensiblement réduite $\left(0,26\right.$ à $\left.0,59 \mathrm{~g} / \mathrm{cm}^{3}\right)$ en regard de celle concernant les feuillus. Il apparaît sur le tableau 1 que le coefficient de variation de $\rho$ passe de 45 p. 100 pour les feuillus à 20 p. 100 dans le cas des résineux, cette très sensible diminution du coefficient de variation est observable pour toutes les caractéristiques élastiques répertoriées.

Les conclusions et commentaires, pour cette population « $\mathrm{R}$ ", quant à l'influence des paramètres physiques $\rho$ et $H$, à travers des régressions linéaires multiples sont similaires à ceux formulés à propos de la population « $F$ " et ne seront pas repris.

Les points représentatifs de chaque caractéristique élastique sont portés en fonction de $\rho$ sur les figures $n^{\circ} 10-18$ et le tableau 4 indique les régressions simples, linéaires et puissances de $\rho$, exprimées en prenant une masse volumique $\bar{\rho}=0,45 \mathrm{~g} / \mathrm{cm}^{3}$, valeur de référence acceptable pour les résineux employés industriellement.

Les qualités des régressions sont significatives au seuil de $1 \%$, à l'exception du module de cisaillement $\mathrm{G}_{\mathrm{RT}}$ pour lequel les coefficients de corrélation indiquent des tests significatifs au seuil de 1 p. 100.

Les coefficients de corrélation relatifs aux lois linéaires sont, systématiquement, légèrement supérieurs à ceux obtenus pour les lois en puissances. De plus, l'étalement des masses volumiques représentatives des bois résineux, étant relativement étroit, l'utilisation de lois puissances n'est pas justifiée.

Les modèles de régressions linéaires seront retenus pour les résineux.

\section{Discussion et conclusion}

L'esprit du travail présenté ici s'inscrit dans la démarche générale suivie par J. Bodig et J.R. Goodman (1973), qui est de rechercher des modèles prévisionnels de lois de comportement élastique tridimensionnel pour les matériaux bois, à travers des corrélations interespèces. Il convient de souligner les compléments et différences qui distinguent les deux approches. En premier lieu, la prise en compte de travaux français récents a permis de compléter la banque de données. Pour les feuillus, l'effectif a été porté de 22 à 43, tandis que pour les résineux, l'effectif est passé de 33 à 37 . Un second point est que les données bibliographiques sont, ici, exploitées en termes des neuf complaisances élastiques $S_{i j}$ comme indiqué au paragraphe 2 , de préférence à 


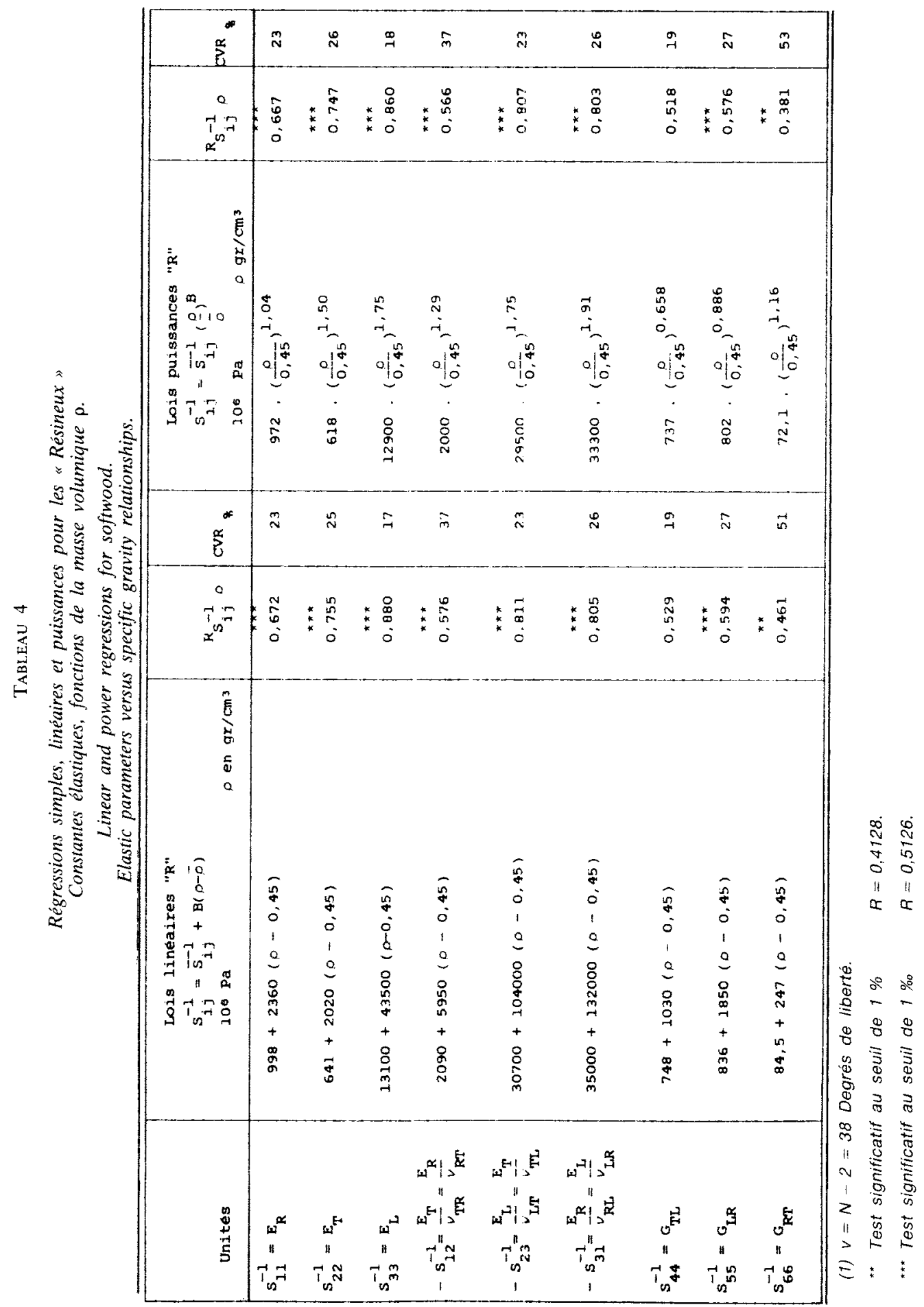


l'utilisation des douze grandeurs techniques qui ne sont, théoriquement, pas indépendantes. En effet, le choix d'un modèle de loi de comportement mécanique élastique linéaire, conduit à l'hypothèse d'existence d'une densité volumique de potentiel élastique, forme quadratique définie positive des composantes par exemple du tenseur des contraintes. Ceci implique la symétrie de la matrice des complaisances élastiques, $\mathrm{S}_{\mathrm{ij}}=\mathrm{S}_{\mathrm{ji}}$.

Dans ces conditions, les mesures, par exemple, des deux modules d'Young $\mathbf{E}_{\mathbf{R}}$ et $E_{T}$ et des deux coefficients de Poisson $\nu_{\mathrm{RT}}$ et $\nu_{\mathrm{TR}}$, donnent quatre informations chiffrées pour déterminer trois grandeurs théoriques indépendantes. Le mode d'exploitation des données, précisé au paragraphe $\mathrm{n}^{\circ} 2$, est ainsi parfaitement justifié. Il a pour conséquence majeure de fournir des régressions significatives entre les complaisances non diagonales et la masse volumique. Ce qui n'est pas le cas lors du traitement direct des coefficients de Poisson, en particulier pour les résineux (Bodig, 1973).

Les deux modèles prévisionnels de loi de comportement élastique proposés pour les bois feuillus sur le tableau 3 (lois puissances) et pour les résineux sur le tableau 4 (lois linéaires) permettent de calculer l'ensemble des termes de la matrice des complaisances élastiques pour un bois dont on connaît la masse volumique $\rho$ mesurée à un taux d'humidité stabilisé au voisinage de 12 p. 100. Sur ces tableaux, un coefficient de variation résiduelle CVR p. 100 pour chaque caractéristique élastique, illustre la part de variabilité du paramètre non expliquée par la masse volumique. L'examen de ces CVR doit être fait en gardant présent à l'esprit que les résultats expérimentaux collectés sont interessences, obtenus au moyen de méthodes expérimentales variées, par différents expérimentateurs. Les prévisions proposées sont particulièrement satisfaisantes. Elles conduisent à des incertitudes du même ordre que celles qui découleraient d'une campagne expérimentale.

En effet, dans le cadre du contrat D.G.R.S.T. $\mathbf{n}^{\circ}$ 81-G-1058 (Pluvinage G., 1985), neuf laboratoires ont été associés à une campagne expérimentale visant à comparer leur méthodologie de mesure de la matrice des complaisances élastiques en travaillant chacun sur plusieurs éprouvettes découpées dans une même grume de hêtre. Les différents lots d'éprouvettes prélevées sur une même grume, échantillonnés par les soins de la Station de Recherches sur la Qualité des Bois du Centre de Recherches forestières de l'INRA (Champenoux), de telle sorte que l'on puisse raisonnablement considérer que l'échantillon moyen était identique pour tous les laboratoires.

Les résultats des essais exécutés par le L.E.M.T.A. (méthode du cube unique), reportés sur le tableau 5, concernent les valeurs moyennes des constantes élastiques mesurées sur 25 cubes de masse volumique moyenne $\rho=0,676 \mathrm{~g} / \mathrm{cm}^{3}(\mathrm{CV}=2,3 \mathrm{p}$. $100)$. Les coefficients de variation observés pour chaque caractéristique sont indiqués. Sur le même tableau 5 sont portées les valeurs prévues par le modèle bois feuillus (lois puissances tableau 3) pour la masse volumique considérée, ainsi que les coefficients de variations résiduelles correspondants.

Certaines rigidités élastiques, particulièrement suivant les directions transverses au fil du bois sont par défaut en regard du modèle. Ceci s'explique en partie du fait que la méthode L.E.M.T.A. suppose actuellement la stabilisation d'un fluage primaire avant l'enregistrement des élongations (quelques dizaines de secondes). Les élongations mesurées sont donc majorées par rapport à une méthode à vitesse de chargement constant, en conséquence les rigidités apparentes sont plus faibles. 


\section{TABI.1NU 5}

Mesures et prévisions des caractéristiques élastiques d'un hêtre.

Experimenal and predicting clastic parameters of beech.

\begin{tabular}{|c|c|c|c|c|c|c|c|c|c|}
\hline & $S_{11}{ }^{1}$ & $S_{21}^{1}$ & $s_{33}{ }^{1}$ & $-S_{12}^{\prime}$ & $-S_{23}{ }^{1}$ & $-S_{31}^{1}$ & $-S_{4+}$ & $-S_{s}{ }^{\prime}$ & $-S_{(s, r}{ }^{\prime}$ \\
\hline \multicolumn{10}{|l|}{ Hêtre } \\
\hline $\begin{array}{l}\text { Valeurs moyennes sur } 25 \text { cubes } \\
\text { L.E.M.T.A. MPa . . . . . . }\end{array}$ & 1550 & 660 & 14900 & 1530 & 22600 & 19700 & 959 & 1490 & 363 \\
\hline$c \vee \% \ldots \ldots \ldots \ldots$ & 7 & 7 & 6 & 11 & 18 & 25 & 9 & 16 & 1.3 \\
\hline \multicolumn{10}{|l|}{ Hetre } \\
\hline $\begin{array}{l}\text { Moctèle feuillus, Prévisions } \\
\rho=0,676 \mathrm{~g} / \mathrm{cm}^{3} \mathrm{MPa}\end{array}$ & 1900 & 1100 & 15000 & 2830 & 32500 & 38700 & 1020 & 1320 & 391 \\
\hline CVR $\% \quad \ldots \ldots \ldots$ & 25 & 37 & 18 & 35 & 35 & 40 & 26 & 31 & 48 \\
\hline \multicolumn{10}{|l|}{ Hêtre } \\
\hline $\begin{array}{l}\text { Moyennes sur } 8 \text { laboratoires } \\
\text { MPa . . . . . . . . . . . }\end{array}$ & 1903 & 890 & 15241 & 2576 & 39419 & 42796 & 1043 & 1400 & 389 \\
\hline$C \vee \% \ldots \ldots \ldots \ldots$ & 22 & 23 & 12 & 41 & 56 & 42 & 20 & 29 & 23 \\
\hline
\end{tabular}

Enfin, les dernières lignes du tableau 5 indiquent les valeurs moyennes et les coefficients de variation de chaque constante élastique du même bois de hêtre, calculées sur un effectif de $\mathrm{N}=8$, correspondants aux résultats affichés par les huit équipes sur dix qui ont, en définitive, transmis des conclusions expérimentales dans le cadre du contrat ci-dessus mentionné. Une lecture comparative de l'ensemble du tableau montre, d'une part que le modèle prévisionnel proposé ici pour les bois feuillus, fournit une loi de comportement élastique tridimensionnelle pour le bois de hêtre étudié, tout à fait satisfaisante; d'autre part, que la multiplication des démarches expérimentales (ici à effectif de 8) n'a pas réduit de façon significative l'intervalle d'incertitude prévu par le modèle.

Il convient de noter que les résultats du contrat relatifs au bois de hêtre ne sont pas inclus dans la banque de données ayant servi à créer les modèles prévisionnels.

En conclusion, le traitement statistique d'une banque de données mécanophysiques, relatives à 43 bois feuillus et 37 bois résineux, permet de proposer deux modèles prévisionnels de loi de comportement élastique en fonction de la masse volumique $\rho \mathrm{du}$ bois considéré à un taux d'humidité $\mathrm{H}$ voisin de 12 p. 100.

L'introduction d'un paramètre supplémentaire, tel que le module longitudinal $E_{\mathrm{l}}$, n'améliore pas de façon significative les régressions multiples.

Les difficultés de mise en auvre d'une méthode expérimentale pour l'identification de la matrice des complaisances élastiques sont telles que les incertitudes sur les résultats obtenus sont du même ordre que celles inhérentes aux modèles proposés. La voie expérimentale ne devrait être choisie que si l'utilisation ultérieure de la matrice complète des complaisances élastiques est pleinement justifiée. 
Dans la majorité des cas, l'ensemble des caractéristiques du comportement élastique tridimensionnel d'un bois ne sera nécessaire que comme données matérielles, dans un problème de mécanique, généralement résolu par une méthode informatisée, type méthode aux éléments finis. L'ingénieur pourra valablement utiliser l'un des modèles élastiques de bois standards proposés et prendre en compte les corrections en masse volumique.

Reçu le 16 juin 1986.

Accepté le 24 décembre 1987.

\section{Remerciements}

- Une part de la collecte et de l'informatisation de la banque de donnée a été assurée grâce à la contribution de Monsieur Sotomayor et de Monsieur Fallou durant leur stage au L.E.M.T.A.

- Cette recherche relevant de la Rhéologie du Bois a été exécutée et a reçu un support financier dans le cadre des activités du G.S. Rhéologie du Bois $n^{\circ} 410082$ du C.N.R.S., et n'a pu se développer qu'en étroite collaboration scientifique avec le C.N.R.F., le C.T.B.A. et le C.T.F.T.

\section{Summary}

\section{Tridimensional elastic behaviour predicting models for hardwood and softwood}

Computed data, including physical properties such as specific gravity $\rho$, moisture content $\mathbf{H}$, and anisotropic elastic properties are available, here for 80 different wood species. Statistical analyses showed significant multiregressions whithin these parameters. This leads to the proposal of two predicting models for the triaxial elastic behaviour for hardwood and softwood. It is then possible to predict the complete clastic compliance matrix for a certain wood whose specific gravity is known, at a nearly 12 p. 100 moisture content. The efficiency of the hardwood elastic model is discussed through experimental results given for the same Beech by cight laboratories.

Key words : Elasticity, anisotropy, hardwood, softwood, predicting models, specific gravity.

\section{Références bibliographiques}

Hearmon R.F.S., 1948. The elasticity of Wood and Plywood. Department of Scientific and Industrial Research. Forest Products Research, Special Report $n^{\circ} 7$, London.

SEICHFPIne J.L., 1980. Mise au point d'une méthode expérimentale destinée à l'identification de la matrice des complaisances élastiques de solides anisotropes: Application aux matériaux bois. Doctorat d'Ingénieur, I.N.P. de Lorraine, Nancy.

Ben Farhat M., 1985. Contribution à la mesure des constantes rhéologiques du bois par compression d'échantillons cubiques. Doctorat de $3 \mathrm{e}$ cycle, I.N.P. de Lorraine, Nancy.

Bodig J., Goodman J.R., 1973. Prediction of elastic parameters for wood. Wood Science, 5 (4), 249-264. 
Guitard D., 1985. Comportement Mécanique du Bois. Dans le matériau bois : propriétés technologie - mise en aeuvre. Ecole d'Hiver sur le Bois à Albe (68), Ed. Arbolor, Nancy, $B^{\mathrm{I}} 29-\mathrm{B}^{\mathrm{I}} 116$.

Guirard D., 1987. Mécanique du matériau hois et composites. Collection nabla. C.E.P.A.D.U.E.S. Editions, Toulouse, 220 pages.

Nepveu G., 1983. Eléments de statistique, INRA-C.N.R.F. Champenoux : Station de Recherches sur la Qualité des Bois.

El AmRı F. Rhéologie du bois (1987). Contribution à là modélisation élastique anisotrope dans le matériau bois-feuillu et résincux. Thèse de Doctorat, I.N.P.L., Nancy.

Gerhards C.C., 1982. Effect of moisture content and temperature on the mechanical properties of Wood: An Analysis of immediate effects. Wood and Fiber, January, 14 (1).

Pluvinage G., 1985. Rapport Contrat D.G.R.S.T. 81 G 1058. Etude critique de la détermination expérimentale des constantes élastiques dans le bois.

Preziosa C., 1982. Méthode de détermination des constantes élastiques du matériau bois par utilisation des ultrasons. Thèse de Docteur Ingénieur, Université d'Orléans.

Stamer J., Sieglerschmidt H., 1933. V.D.I. page 503. Zeitschrift des Vereines Deutscher Ingenieure, bd 77, Berlin, $\mathrm{Nr} 19$.

Kollmann F.F.P., Core W.A. Principales of Wood Science and Technology. Volume I. Solid Wood, 294-296. Springer Verlag, Berlin, Heidelberg, New York, Tokyo.

Launay J. E.S.E.M. Orléans, Communication personnelle. 